\title{
Excess Electron Attachment to Disulfide-Bridged L,L-Cystine. An ab Initio Study
}

\author{
Agnieszka Sawicka, ${ }^{\dagger, \ddagger}$ Piotr Skurski, ${ }^{*}, \dagger, \ddagger$ and Jack Simons ${ }^{\ddagger}$ \\ Department of Chemistry, University of Gdańsk, Sobieskiego 18, 80-952 Gdańsk, Poland, and Henry Eyring \\ Center for Theoretical Chemistry, Department of Chemistry, University of Utah, Salt Lake City, Utah 84112
}

Received: October 23, 2003; In Final Form: February 19, 2004

\begin{abstract}
The possibility of excess electron binding to cystine (consisting of two L-cysteine molecules linked via a disulfide bridge) in the gas-phase was studied at the second-order Møller-Plesset perturbation theory (MP2) level using the $6-31+\mathrm{G}^{* *}+6$ (sp) basis sets. Several geometrically stable conformers and tautomers were found on the potential energy surfaces (PES) of both the neutral and anionic species. The most stable neutral isomer has proven to (i) involve two canonical rather than zwitterionic cysteine monomers, (ii) possess no inter-monomer hydrogen bonds, and (iii) exhibit an extended structure due to the presence of four intramonomer hydrogen bonds. It has also been found that most neutral isomers are capable of excess electron binding to form geometrically and electronically stable anions of dipole-bound nature. The electron binding energies for these anions span a wide region of $0.0004-0.947 \mathrm{eV}$ (depending on the neutral parent molecule). In addition, several cystine-based anions were found at geometries where the neutral species are not stable. The latter anions gain stability from their large electron binding energies (they bind an excess electron by $0.488-1.975 \mathrm{eV})$.
\end{abstract}

\section{Introduction}

It is well-known that covalent disulfide bonds are very important determinants of the shapes of peptides and proteins because the $\mathrm{S}-\mathrm{S}$ bonds between cysteine building blocks stabilize folded conformations. ${ }^{1}$ Moreover, the structures and properties of molecules containing sulfur-sulfur bonds have been of interest for decades since the disulfide linkage plays an important role in enzyme and antibiotic structure stabilization and in the biological activity of molecular systems. It has also been shown that protein disulfide radical anions are stable in aqueous solutions, ${ }^{2}$ and the reduction of only one disulfide bridge does not have to modify the protein conformation. ${ }^{3}$

Cysteine (Cys) as a sulfur-containing amino acid, the cysteine dimer Cys-Cys (involving two cysteine molecules linked with the $\mathrm{S}-\mathrm{S}$ bond), cystine-containing peptides, and cystine-based cyclic oligoureas have been the subject of experimental and theoretical studies ${ }^{4-8}$ recently. In particular, Dahaoui et al. studied the electron charge density of L-cystine using X-ray diffraction techniques and concluded that the almost tetrahedral distribution of the valence shell charge concentration of the sulfur atoms suggests $\mathrm{sp}^{3}$ hybridization. ${ }^{4}$ In addition, they showed that the structure of L-cystine in crystals is stabilized by hydrogen bonds linking molecules via the $\mathrm{COO}^{-}$and $\mathrm{NH}_{3}{ }^{+}$ groups. ${ }^{4}$ Ross and Burrows studied nickel complexes of both cysteine and cystine-containing peptides investigating the spontaneous formation of disulfide-bridged dimers, ${ }^{6}$ whereas Ranganathan et al. proposed and examined a new class of hydrogen bonding electroneutral anion receptors (cystine-based oligoureas). ${ }^{5,7}$ Most recently, Hameka et al. studied the fluorescence of the neutral cystine by comparing the configuration interaction with single-excitations configuration interaction theoretical results to the results of their experimental measurements of the UV absorption and fluorescence spectra. ${ }^{8}$

\footnotetext{
* To whom correspondence should be addressed. E-mail: piotr@ chem.univ.gda.pl.

†niversity of Gdańsk.

University of Utah.
}

Even though the number of publications devoted to cysteinecontaining compounds seems large, it is surprising that, to the best of our knowledge, there have been no papers dealing with the possibility of an excess electron binding to cystine published in the literature thus far. The only attempt to study the nondissociative electron capture by the species which mimic the disulfide-bridged cystine containing one $\mathrm{S}-\mathrm{S}$ bond is that reported by Carles et al. and investigating the nondissociative electron capture by a series of saturated disulfides given by the $\mathrm{R}-\mathrm{S}-\mathrm{S}-\mathrm{R}$ formula (where R stands for $\mathrm{CH}_{3}, \mathrm{C}_{2} \mathrm{H}_{5}$, and $\mathrm{C}_{3} \mathrm{H}_{7}$ ) ${ }^{9}$ The neutral model systems RS-SR were examined using Rydberg electron-transfer spectroscopy (RETS), negative ion photoelectron spectroscopy, and computational techniques (MP2 and DFT methods). It has been shown that the saturated disulfides form stable anions but do not capture thermal (nearly zero energy) electrons when isolated. As Carles and co-workers demonstrated, such capturing is possible only when these molecules are imbedded in clusters.

Studying the interaction with an excess electron is a fundamental step in constructing a knowledge base that will ultimately be of use in all cases. The main goal of the present work is to provide information lacking in the literature about excess electron binding to various isomers of cystine. Since cystine consists of two cysteine units, we must consider several possible tautomers, such as canonical-canonical, canonical-zwitterionic, and so on. In particular, by using ab inito Hartree-Fock and second-order Møller-Plesset perturbation theory treatments, we examine the lowest energy isomers of the neutral cystine and for each locally geometrically stable structure we investigate the possibility of forming an electronically stable anion. In addition, we study the anionic potential energy surface to search for stable anion structures that do not correspond to locally geometrically stable neutral parents.

\section{Methods}

The ground-state potential energy surfaces of the neutral and anionic L,L-cystine (i.e., consisting of two L-cysteine molecules 
linked via the $\mathrm{S}-\mathrm{S}$ bond) were studied at the Hartree-Fock self-consistent field level of theory, whereas the final estimates of the relative energies were calculated at the second-order Møller-Plesset perturbation theory (MP2) ${ }^{10}$ level. Because the methods we used are based on an unrestricted Hartree-Fock (UHF) starting point, it is important to make sure that little if any artificial spin contamination enters into the final wave functions. We computed the expectation value $\left\langle S^{2}\right\rangle$ for species studied in this work and found values of $0.7500-0.7505$ in all anion cases. Hence, we are certain that spin contamination is not large enough to significantly affect our findings.

The electron binding energies $(D)$ were calculated using a supermolecular approach (i.e., by subtracting the energies of the anion from those of the neutral). This approach requires the use of size-extensive methods for which we have employed second-order Møller-Plesset perturbation theory. In addition, $D$ was analyzed within the perturbation framework designed for dipole-bound anions and solvated electrons described previously by Gutowski and Skurski. ${ }^{11}$

The simplest theoretical approach to estimate $D$ is based on Koopmans' theorem (KT). ${ }^{12}$ The KT binding energy $\left(D^{\mathrm{KT}}\right)$ is the negative of the energy of the relevant unfilled orbital obtained from a Hartree-Fock self-consistent field (SCF) calculation on the neutral molecule. This is a static approximation to the electron binding energy, which neglects both orbital relaxation and electron correlation effects. These effects were taken into account by performing SCF and MP2 calculations for the neutral and the anion.

The polarization of the neutral host $(N)$ by the excess electron and the effect of back-polarization are taken into account when the SCF calculation is performed for the anion $(A)$, and the accompanying induction effects on $D$ are given by

$$
\Delta D_{\text {ind }}^{\mathrm{SCF}}=D^{\mathrm{SCF}}-D^{\mathrm{KT}}
$$

where

$$
D^{\mathrm{SCF}}=E_{\mathrm{N}}^{\mathrm{SCF}}-E_{\mathrm{A}}^{\mathrm{SCF}}
$$

and $E_{\mathrm{N}}^{\mathrm{SCF}}$ and $E_{\mathrm{A}}^{\mathrm{SCF}}$ stand for the SCF energies of the neutral and the anion, respectively.

The dispersion interaction between the loosely bound electron and $N$ was extracted from the MP2 contribution to $D$. The dispersion term is a second-order correction with respect to the fluctuation-interaction operator and it is approximated here by $\Delta D_{\text {disp }}^{\mathrm{MP2}}$, which takes into account proper permutational symmetry for all electrons in the anion

$$
\epsilon_{\mathrm{disp}}^{(02)} \approx \sum_{a \in N} \sum_{r<s} \frac{\left|\left\langle\phi_{\mathrm{a}} \phi_{\mathrm{mbe}}|| \phi_{\mathrm{r}} \phi_{\mathrm{s}}\right\rangle\right|^{2}}{e_{\mathrm{a}}+e_{\mathrm{mbe}}-e_{\mathrm{r}}-e_{\mathrm{s}}}=-\Delta D_{\mathrm{disp}}^{\mathrm{MP} 2}
$$

where $\phi_{\mathrm{a}}$ and $\phi_{\mathrm{mbe}}$ are spin orbitals occupied in the unrestricted Hartree-Fock anion wave function, $\phi_{\mathrm{r}}$ and $\phi_{\mathrm{s}}$ are unoccupied orbitals, and the $e$ 's are the corresponding orbital energies. The subscript mbe denotes the multipole (dipole in this case) bound electron's spin-orbital.

The total MP2 contribution to $D$ defined as

$$
\Delta D^{\mathrm{MP} 2}=D^{\mathrm{MP} 2}-D^{\mathrm{SCF}}
$$

is naturally split into dispersion and nondispersion terms

$$
\Delta D^{\mathrm{MP} 2}=\Delta D_{\mathrm{disp}}^{\mathrm{MP} 2}+\Delta D_{\mathrm{no}-\text { disp }}^{\mathrm{MP}}
$$
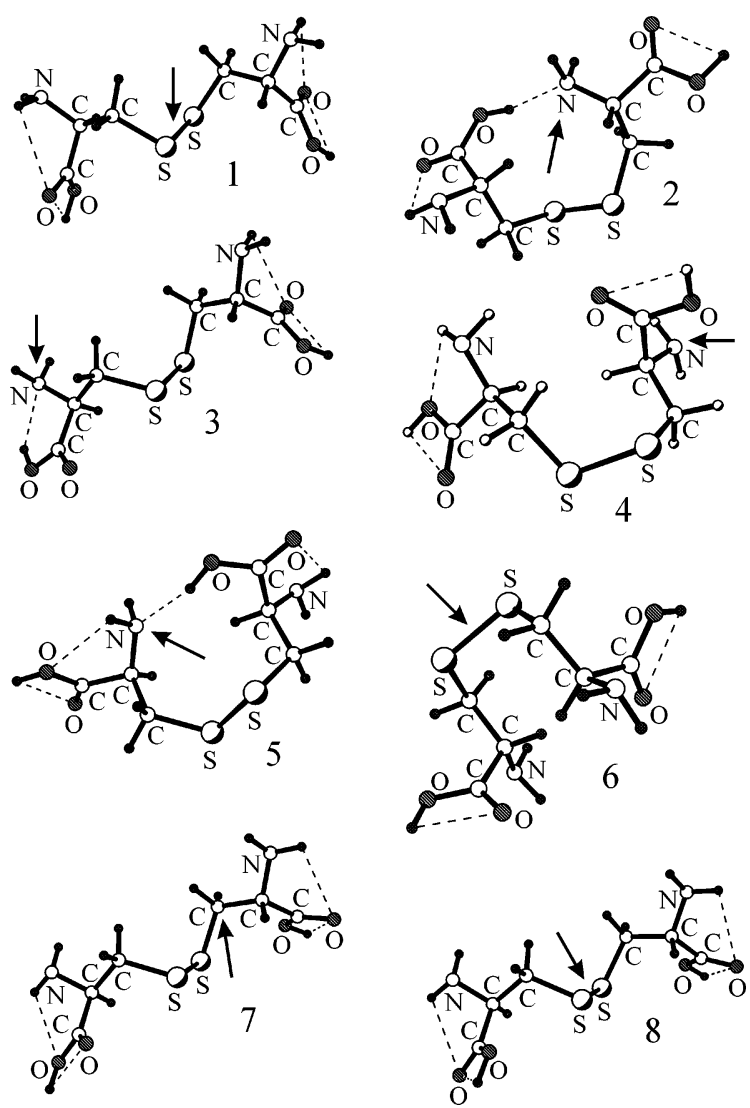

Figure 1. Equilibrium structures corresponding to the neutral minima $\mathbf{1 - 8}$ of cystine studied in this work (hydrogen bonds are indicated with dashes).

with the latter dominated by the correlation correction to the static Coulomb interaction between the loosely bound electron and the charge distribution of $N$.

Since the electron binding energies for anionic species considered in this work were obtained at the MP2 level, the values of $D^{\mathrm{MP} 2}$ produce our final estimates of $D$ for each system studied.

The diffuse character of the orbital describing the loosely bound electron necessitates the use of extra diffuse basis functions having very low exponents. ${ }^{13}$ In addition, the basis sets chosen to describe the neutral molecular host should be flexible enough to (i) accurately describe the static charge distribution of the neutral and (ii) allow for polarization and dispersion stabilization of the anion upon electron attachment. The geometry optimization calculations and the vibrational frequency calculations were performed with the $6-31+\mathrm{G}^{* *}$ basis set $^{14,15}$ supplemented with a $5(\mathrm{sp})$ set of diffuse functions centered either in the center of the $\mathrm{S}-\mathrm{S}$ disulfide bridge or on one of the atoms in the vicinity of the positive end of the molecular dipole (as indicated in Figures 1-3 where the additional basis functions centering sites are marked with an arrow).

The evaluation of the electron binding energies was performed with the $6-31+\mathrm{G}^{* *}$ basis set supplemented with a $6(\mathrm{sp})$ set of diffuse functions centered as described above. The $6-31+\mathrm{G}^{* *}$ basis set was chosen since we earlier showed its usefulness in describing multipole-bound anions compared to other commonly used one-electron basis sets. ${ }^{13}$ In particular, the $6-31+\mathrm{G}^{* *}$ basis set produces reliable estimates of the electron binding energies for such species which are only slightly underestimated comparing to those obtained with larger (and thus more time-expensive) basis sets as aug-cc-pVDZ basis, ${ }^{16}$ for example. ${ }^{13}$ The extra 

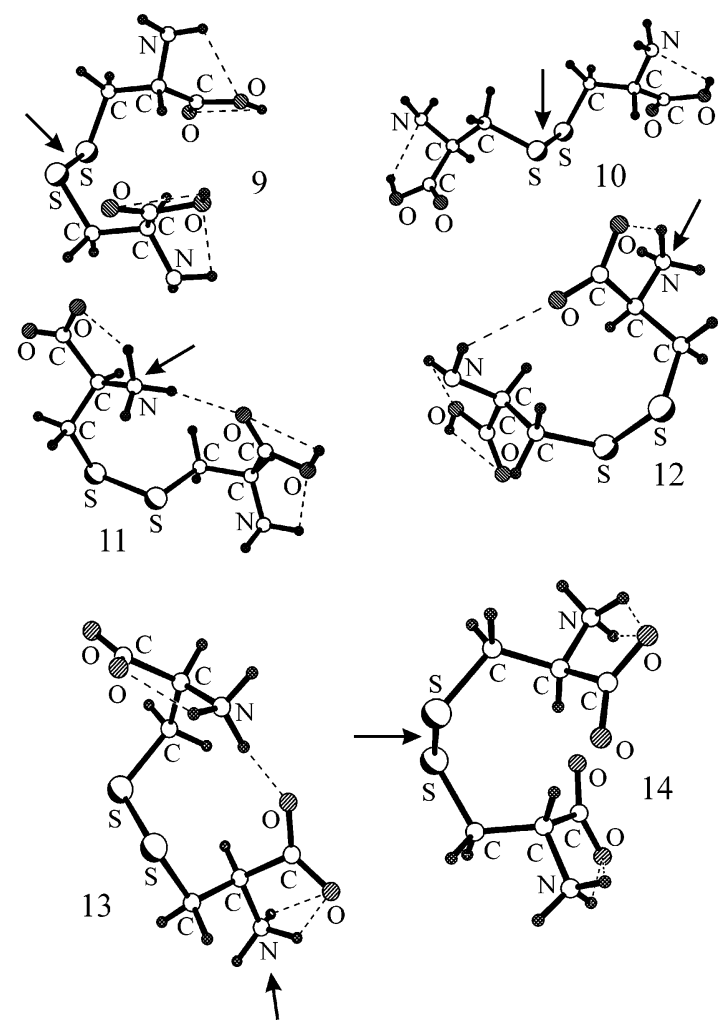

Figure 2. Equilibrium structures corresponding to the neutral minima 9-14 of cystine studied in this work (hydrogen bonds are indicated with dashes).
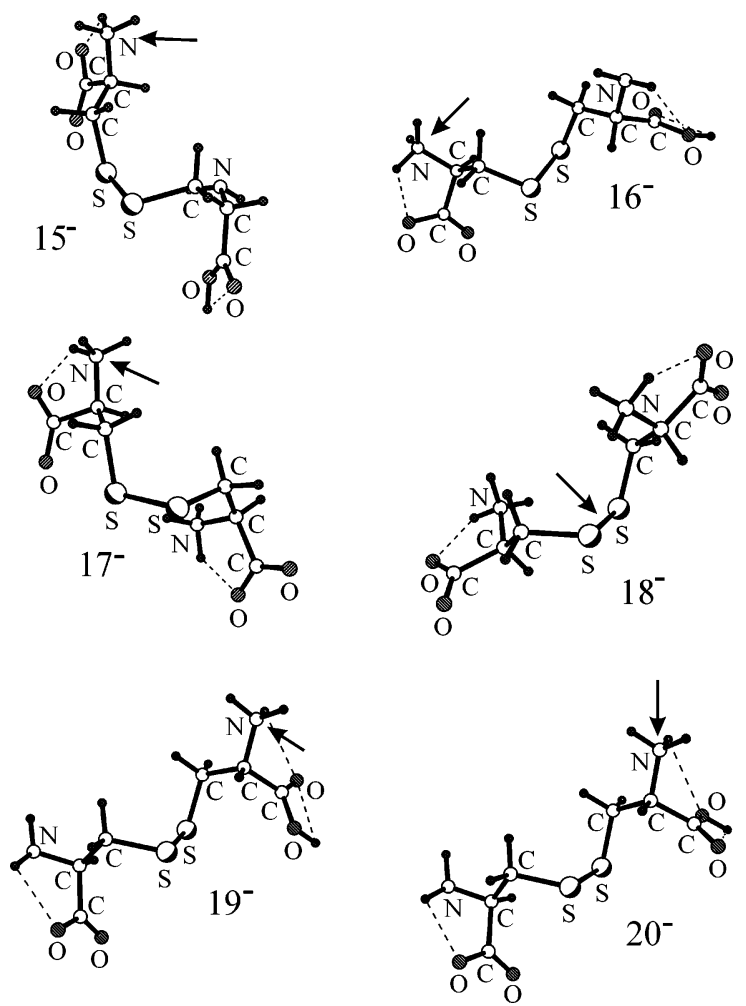

Figure 3. Equilibrium structures corresponding to the anionic minima $\mathbf{1 5}^{-}-\mathbf{2 0}^{-}$of cystine studied in this work (hydrogen bonds are indicated with dashes).

diffuse functions share exponent values and we used eventempered ${ }^{17}$ six-term $\mathrm{sp}$ basis sets (for electron binding energy evaluations). The geometric progression ratio was equal to 5.0, ${ }^{18}$ and we started to build up the exponents of the extra diffuse functions from the lowest exponent of the same (i.e., sp) symmetry included in $6-31+\mathrm{G}^{* *}$ basis set designed for sulfur. As a consequence, we achieved the lowest exponent of 2.5920 $\times 10^{-6}$ a.u., for the sp symmetry.

All calculations were performed with the Gaussian 98 program, ${ }^{19}$ whereas the three-dimensional plots of molecular orbitals were generated with the MOLDEN program. ${ }^{20}$

\section{Results}

3.1. Neutral Isomers of Disulfide-Bridged Cystine and Their Relative Energies. The ground-state potential energy surface (PES) of the neutral cystine is rather complicated because of the large number of conformers (mostly rotamers) that might correspond to local minima. Therefore, we had to use our chemical intuition while proposing the structures that could represent locally geometrically stable isomers. While doing so, we focused on the structures that are derived from the typical disulfide bridged system by rotating various functional groups around the single $\mathrm{S}-\mathrm{S}, \mathrm{S}-\mathrm{C}, \mathrm{C}-\mathrm{C}, \mathrm{C}-\mathrm{O}$, and $\mathrm{C}-\mathrm{N}$ bonds. In particular, we tried to generate (as starting geometries for further geometry optimizations) the structures in which the maximum possible number of hydrogen bonds is present. Moreover, we considered not only cystine isomers that consist of two canonical (i.e., containing $-\mathrm{NH}_{2}$ and $-\mathrm{COOH}$ groups) monomers but also those involving the zwitterionic fragments (i.e., in which the proton has been transferred from $-\mathrm{COOH}$ to $-\mathrm{NH}_{3}$ group leading to $-\mathrm{COO}^{-}$and $-\mathrm{NH}_{3}{ }^{+}$, respectively). As a consequence, a relatively large set of starting structures has been generated and the geometry optimization procedure (followed by harmonic vibrational analysis) has been employed for each of them to verify whether the resulting converged structures correspond to local minima on the neutral PES. This led us to 14 isomers of the neutral disulfide-bridged cystine (see Figures 1 and 2 and Table 1S), each of which is a minimum on the PES (i.e., is characterized by all real vibrational frequencies).

The isomers labeled $\mathbf{1 - 1 0}$ are of CC type (where CC stands for canonical-canonical) which means that they involve two canonical cysteine monomers linked via an $\mathrm{S}-\mathrm{S}$ bridge. Isomer $\mathbf{1}$ is the lowest energy neutral species, and its structure is rather stretched due to the fact that all four hydrogen bonds that are formed in $\mathbf{1}$ (see Figure 1) are intra-monomer H-bonds that link the centers belonging to the same monomer only. In other words, the inter-monomer hydrogen bonds (involving the atoms belonging to both monomers and causing the whole structure to be more compact) are absent. The $\mathrm{C}-\mathrm{S}-\mathrm{S}-\mathrm{C}$ dihedral angle involving the disulfide bridge is close to $90^{\circ}$ for $\mathbf{1}\left(86.75^{\circ}\right)$, as it is the case for all other $(\mathbf{2}-\mathbf{1 4})$ structures. Since $\mathbf{1}$ is the global minimum on the ground-state neutral PES, we decided to provide the energies of the other isomers as relative numbers (with respect to the energy of $\mathbf{1}$ which is taken as zero) to show the isomer-isomer energy gaps in a more convenient way.

The relative energies of the isomers labeled $\mathbf{2 - 1 0}$ are in the $0.000-0.271 \mathrm{eV}(0.0-6.2 \mathrm{kcal} / \mathrm{mol})$ range (see Table 1$)$. Isomer 2 (see Figure 1) is $0.088 \mathrm{eV}(2.0 \mathrm{kcal} / \mathrm{mol})$ higher in energy than 1 and this energy gap (even though it is not large) indicates that one might expect isomer $\mathbf{1}$ to be the lowest energy structure even when a more sophisticated theoretical treatment is applied. On the other hand, small energy differences among all CCtype 1-10 isomers indicate that it is likely that the energy ordering of tautomers could be altered if different theoretical treatments (i.e., method and basis sets) were used. This observation is valid especially for those structures whose energies differ by less than $0.015 \mathrm{eV}(0.2 \mathrm{kcal} / \mathrm{mol})$, as it is the 
TABLE 1: Relative Energies (in $\mathrm{eV}$ ) of Various Neutral and Anionic Cystine Isomers Studied in This Work (with Respect To the Most Stable Neutral Tautomer 1 Whose Energy Was Taken as Zero) Calculated at the MP2 Level with the 6-31+G**+6(sp) Basis Sets ${ }^{a}$

\begin{tabular}{cccrcccr}
\hline \multicolumn{3}{c}{$\begin{array}{c}\text { relative } \\
\text { species }\end{array}$} & energy & \multicolumn{1}{c}{$\mu^{\mathrm{SCF}}$} & \multicolumn{1}{c}{$\mu^{\mathrm{MP} 2}$} & \multicolumn{3}{c}{ species } & energy & $\mu^{\mathrm{SCF}}$ & $\mu^{\mathrm{MP} 2}$ \\
\hline $\mathbf{1}$ & $0.000^{b}$ & 0.329 & 0.101 & $\mathbf{1}^{-}$ & & unstable \\
$\mathbf{2}$ & 0.088 & 6.648 & 6.278 & $\mathbf{2}^{-}$ & 0.061 & 6.807 & 6.424 \\
$\mathbf{3}$ & 0.128 & 5.272 & 4.753 & $\mathbf{3}^{-}$ & 0.109 & 5.389 & 4.857 \\
$\mathbf{4}$ & 0.135 & 4.474 & 3.966 & $\mathbf{4}^{-}$ & 0.131 & 4.528 & 4.013 \\
$\mathbf{5}$ & 0.163 & 4.679 & 4.672 & $\mathbf{5}^{-}$ & 0.145 & 4.816 & 4.794 \\
$\mathbf{6}$ & 0.177 & 0.213 & 0.426 & $\mathbf{6}^{-}$ & & unstable \\
$\mathbf{7}$ & 0.191 & 3.864 & 3.119 & $\mathbf{7}^{-}$ & 0.191 & 3.884 & 3.136 \\
$\mathbf{8}$ & 0.224 & 3.069 & 2.334 & $\mathbf{8}^{-}$ & & unstable \\
$\mathbf{9}$ & 0.229 & 4.447 & 4.088 & $\mathbf{9}^{-}$ & 0.228 & 4.554 & 4.179 \\
$\mathbf{1 0}$ & 0.271 & 8.421 & 7.679 & $\mathbf{1 0}^{-}$ & 0.147 & 8.697 & 7.924 \\
$\mathbf{1 1}$ & 0.581 & 12.515 & 11.722 & $\mathbf{1 1}^{-}$ & 0.382 & 13.056 & 12.242 \\
$\mathbf{1 2}$ & 0.821 & 10.174 & 9.696 & $\mathbf{1 2}^{-}$ & 0.319 & 11.781 & 11.191 \\
$\mathbf{1 3}$ & 1.082 & 16.514 & 15.699 & $\mathbf{1 3}^{-}$ & 0.278 & 19.139 & 18.222 \\
$\mathbf{1 4}$ & 1.128 & 3.965 & 3.662 & $\mathbf{1 4}^{-}$ & 1.125 & 4.059 & 3.662 \\
& & & & $\mathbf{1 5}^{-}$ & 0.558 & 10.131 & 9.375 \\
& & & & $\mathbf{1 6}^{-}$ & 0.675 & 11.851 & 10.879 \\
& & & & $\mathbf{1 7}^{-}$ & 0.836 & 15.748 & 14.753 \\
& & & & $\mathbf{1 8}^{-}$ & 0.998 & 4.841 & 4.653 \\
& & & & $\mathbf{1 9}^{-}$ & 1.529 & 33.499 & 32.815 \\
& & & & $\mathbf{2 0}^{-}$ & 1.583 & 35.169 & 34.281
\end{tabular}

${ }^{a}$ The SCF and MP2 dipole moments $(\mu)$ for the neutral species are given for each geometrically stable species. ${ }^{b}$ The energy of the most stable neutral cystine isomer is -1439.7662688 au (as calculated at the MP2/6-31+G**+6(sp)//SCF/6-31+G**+5(sp) level).

case for structures $\mathbf{3}$ and $\mathbf{4}$, structures $\mathbf{5}$ and $\mathbf{6}$, and structures $\mathbf{8}$ and 9 (see Table 1). In those cases, one cannot judge whether one isomer is more stable than another (in the gas phase) since the MP2 method does not offer such an accuracy for the relative energies.

Structures $\mathbf{1 1}$ and $\mathbf{1 2}$ correspond to ZC isomers (where ZC stands for Zwitterionic-Canonical) which means that they involve one canonical cysteine monomer and one zwitterionic monomer linked via an $\mathrm{S}-\mathrm{S}$ bridge (see Figure 2). Relative energies for these two isomers are in the $0.581-0.821 \mathrm{eV}$ (13.4-18.9 $\mathrm{kcal} / \mathrm{mol}$ ) range (see Table 1) with respect to the most stable structure 1 . Therefore, we conclude that replacing one of the canonical monomers in CC-type isomer of cystine with the zwitterionic monomer raises the total energy of the isomer by at least $0.3 \mathrm{eV}(7 \mathrm{kcal} / \mathrm{mol})$ which is simply the energy gap between the highest-energy $\mathrm{CC}$ isomer (10) and the lowest energy ZC structure (11).

The isomers labeled $\mathbf{1 3}$ and $\mathbf{1 4}$ correspond to $\mathrm{ZZ}$ isomers (where ZZ stands for Zwitterionic-Zwitterionic) which means that they involve two zwitterionic cysteine monomers linked via an $\mathrm{S}-\mathrm{S}$ bridge (see Figure 2). Relative energies (i.e., with respect to 1) for these two structures are in the 1.082-1.128 eV (25.0-26.0 kcal/mol) range (see Table 1). Therefore, we conclude that replacing the canonical monomer in ZC-type cystine with the zwitterionic monomer raises the total energy of the isomer by at least $0.26 \mathrm{eV}(6 \mathrm{kcal} / \mathrm{mol})$ which is the energy gap between the highest-energy $\mathrm{ZC}$ isomer (12) and the lowest energy ZZ structure (13).

Surprisingly, neither the number of hydrogen bonds nor the overall "shape" of the molecule (i.e., compact vs stretched) seems to determine the relative energy of the neutral system. However, we note that the structures in which the carbonyl oxygen atoms are involved in the hydrogen bonds are preferred. In particular, this is the case for isomers $\mathbf{1}$ and $\mathbf{2}$ (where two such bonds are present as shown in Figure 1). Certainly, the largest number of hydrogen bonds (four) can be found for the two lowest isomers (1 and $\mathbf{2}$ ), although four $\mathrm{H}$-bonds are also present in the higher energy isomers (e.g., $\mathbf{5}$ and $\mathbf{7}$, see Figure $1)$.

We are aware of the fact that the PES of the neutral disulfidebridged cystine is very complicated and it is not likely to scan all of the possible minima without employing additional techniques as genetic algorithms, for example. However, our goal was to explore the possibility of forming electronically stable cystine anions rather than providing accurate relative energies of the neutral cystine isomers.

3.2. Polarity of the Neutral Cystine Isomers. The focus of this work is on the possibility of forming electronically stable anionic states supported by various cystine isomers. Because cystine is a valence-saturated closed-shell neutral molecule (i.e., it contains no vacant or half-filled molecular orbitals) and is not expected to form stable valence anionic states, we conclude that the only electronically stable anions supported by this species should be those exhibiting either dipole-bound or Rydberg nature. It is for this reason that we consider the polarity of the neutral cystine isomers, manifested by their dipole moments, as an important feature. Since it is known that a neutral closed-shell molecule possessing dipole moment that is larger than 2.5-3.0 D is capable of forming electronically stable dipole-bound anions having significant binding energies, we analyze the polarity of the isomers studied in this work judging their ability to support such states.

The dipole moment of the lowest energy neutral cystine isomer (1) is only $0.101 \mathrm{D}$ (as calculated from the MP2 charge density, see Table 1); thus, isomer $\mathbf{1}$ is not expected to support electronically stable anionic state of dipole-bound nature. The next four neutral isomers in the relative energy scale (i.e., structures 2-5) are more polar and their dipole moments are in the range of 3.97-6.28 D (see Table 1), even though each of these structures consists of two canonical cysteine monomers. We expect these isomers to form electronically stable dipolebound anions, as is also the case for structures $\mathbf{7 , 9}$, and $\mathbf{1 0}$, whose dipole moments were found to be 3.12, 4.09, and 7.68 $\mathrm{D}$, respectively. By contrast, isomers $\mathbf{6}$ and $\mathbf{8}$ possess the dipole moments ( 0.43 and $2.33 \mathrm{D}$, respectively) that are too small to support dipole-bound anionic states (see Table 1).

The ZC and ZZ isomers (i.e., consisting of either one zwitterionic and one canonical or two zwitterionic cysteine fragments, respectively) labeled 11-14 in this contribution are much more polar having dipole moments in the 9.70-15.70 D range, except isomer $\mathbf{1 4}$ for which the value of $\mu$ is $3.66 \mathrm{D}$. Stronger polarity of the $\mathrm{ZC}$ and $\mathrm{ZZ}$ isomers is obviously caused by the presence of one or two $\left(-\mathrm{NH}_{3}\right)^{+}$sites and the corresponding one or two $(-\mathrm{COO})^{-}$groups. The relatively large separation between each $\left(-\mathrm{NH}_{3}\right)^{+} /(-\mathrm{COO})^{-}$pair causes both (i) an increase of the total energy and (ii) an increase of the excess electron attachment energy. Although the former effect is strongly destabilizing (it causes the $\mathrm{ZC}$ and $\mathrm{ZZ}$ isomers to be much higher in energy than the global minimum 1), the latter is stabilizing in a sense that it leads to larger electron binding energy of a daughter anion.

We conclude that 7 out of 10 cystine CC isomers as well as all four $\mathrm{ZC}$ and $\mathrm{ZZ}$ cystine structures should be capable of an excess electron binding. In addition, we are aware of the fact that, except those supported by the $\mathbf{2 - 5}, \mathbf{7}$, and 9-14 neutral parents, there could also be other cystine anionic structures that correspond to local minima on the ground-state anionic potential energy surface. Such minima would not correspond to any geometrically stable neutral parent but develop on the anionic PES due to the large vertical electron binding energy character- 

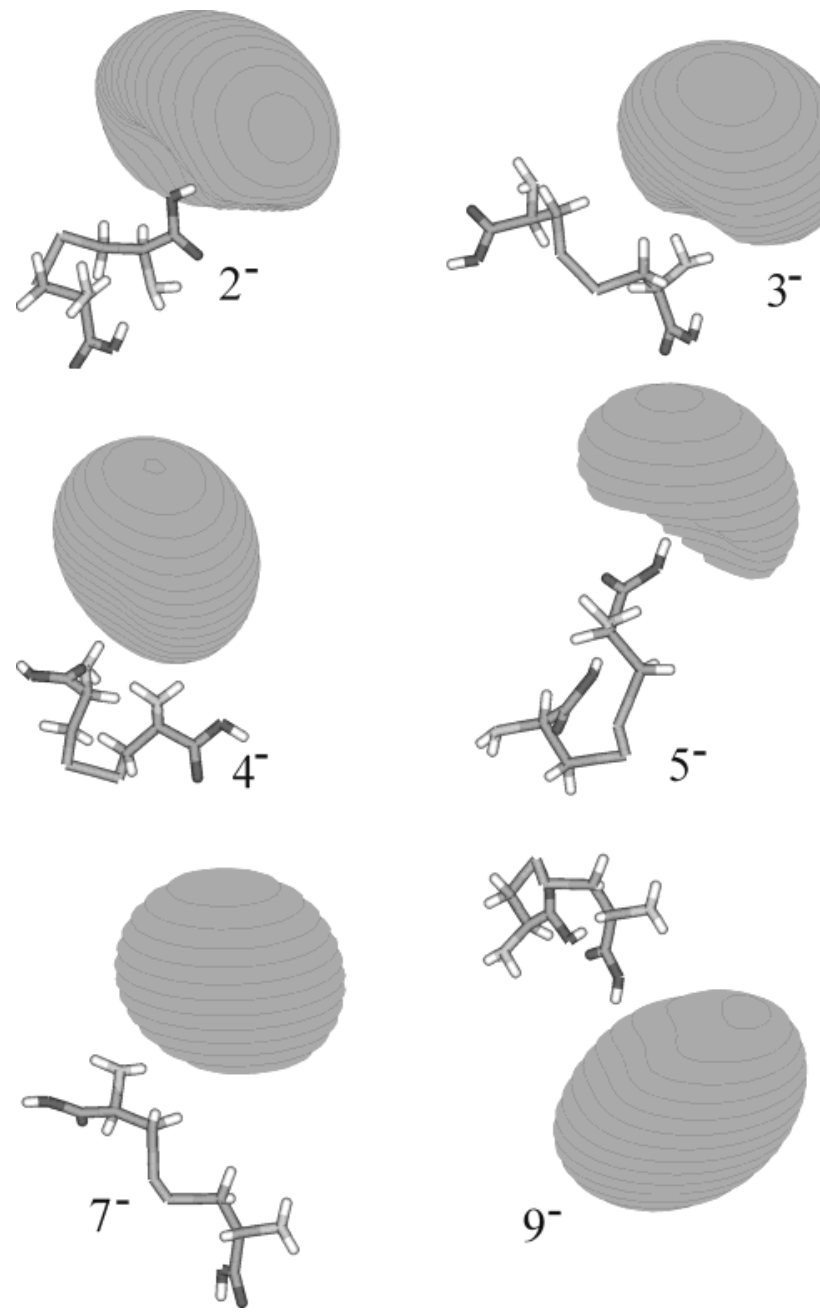

Figure 4. Singly occupied molecular orbital (SOMO) holding the excess electron in the ground electronic states of anions supported by $\mathbf{2}-\mathbf{5}, \mathbf{7}$, and $\mathbf{9}$ cystine isomers.

izing certain geometrical structure. We discuss these issues in the following sections.

3.3. Anions Formed by Various Isomers of Neutral Cystine and Their Relative Energies. Having identified isomers 1-14 of the neutral disulfide-bridged cystine, we move on to the discussion of the various anions that can be formed by these species. In Table 1, the relative energies of the anionic systems are given (with respect to the neutral global minimum 1) but only for those species that are vertically electronically stable. As indicated in Table 1, the anion based on the most stable neutral (1) is not electronically stable because of the small polarity of $\mathbf{1}$ (manifested by its dipole moment of $0.101 \mathrm{D}$ ). We observe the same situation (i.e., the lack of electronic stability of their daughter anions) for two other isomers (6 and 8) whose dipole moments are also small (not exceeding $2.5 \mathrm{D}$, see Table 1). Even though the other low-energy neutral isomers (i.e., 2, 3, 4, 5, 7, and 9) do support stable anionic states, their polarity is small (the corresponding dipole moments are in the range of $3.12-6.28 \mathrm{D}$ ) and thus the resulting vertical electron binding energies are not large enough to render these anions adiabatically stable (i.e., with respect to the neutral global minimum 1). It should also be noted that the equilibrium geometries of the $\mathbf{2}^{-}, \mathbf{3}^{-}, \mathbf{4}^{-}, \mathbf{5}^{-}, \mathbf{7}^{-}$, and $\mathbf{9}^{-}$anions are very similar to the geometries of their corresponding neutral parents and they are depicted in Figures 4 and 5 where the singly occupied molecular orbitals holding the excess electrons in each of these anions are also depicted (see also Table $1 \mathrm{~S}$, in the
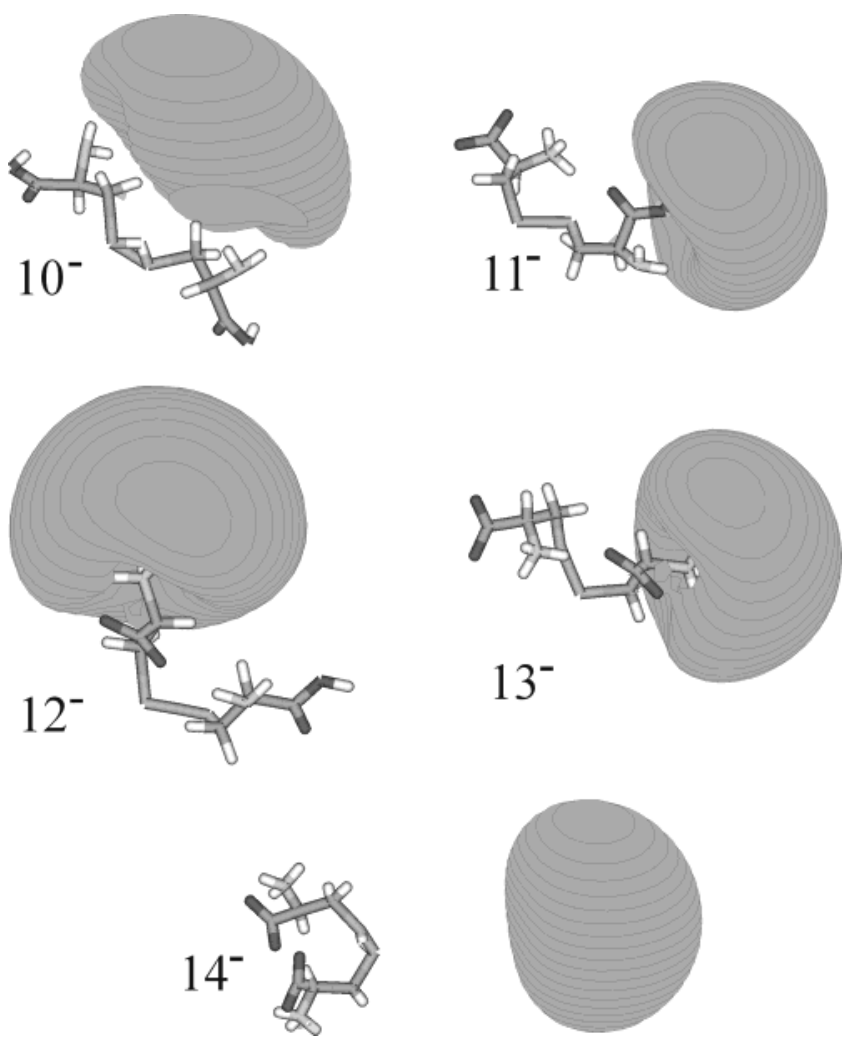

Figure 5. Singly occupied molecular orbital (SOMO) holding the excess electron in the ground electronic states of anions supported by 10-14 cystine isomers.

Supporting Information, for geometrical coordinates of these systems). It can be seen that in each anionic case the extra electron is localized primarily outside the molecular framework on the positive side of the molecular dipole, as it has been previously observed for other dipole-bound anions. ${ }^{11,13,18,21-35}$

The polarity of the CC neutral tautomer $\mathbf{1 0}$ and of the ZC and $\mathrm{ZZ}$ tautomers $\mathbf{1 1 - 1 3}$ is larger and these systems (in their neutral local minimum energy structures) exhibit dipole moments in the $7.68-15.70 \mathrm{D}$ range (see Table 1 ). This causes the excess electron binding energies for their daugther anions to be larger (from 0.11 to $0.95 \mathrm{eV}$ ) as indicated in Table 2 . However, even such large $D$ values (characterizing the anions formed by an excess electron attachment to 10-13) do not render these anions thermodynamically stable with respect to the neutral global minimum $\mathbf{1}$ plus a free electron. This is because the energy difference between any of the 10-13 neutral species and $\mathbf{1}$ is always larger than the energy lowering due to the excess electron attachment to this system (see Tables 1 and 2). For example, the energy difference between the neutral species $\mathbf{1 0}$ and $\mathbf{1}$ is $0.271 \mathrm{eV}$, whereas the electron binding energy calculated for $\mathbf{1 0}^{-}$is $0.111 \mathrm{eV}$. The corresponding values for isomers $\mathbf{1 1 - 1 3}$ are $0.581 \mathrm{eV}$ vs $D=0.226 \mathrm{eV}$ (for 11), $0.821 \mathrm{eV}$ vs $D=0.599 \mathrm{eV}$ (for 12), and $1.082 \mathrm{eV}$ vs $D=$ $0.947 \mathrm{eV}$ (for 13), where the former numbers (for each pair) indicate the instability of a given neutral with respect to $\mathbf{1}$, whereas the latter numbers are the excess electron binding energies for the corresponding anions.

To complete the list of the neutral cystine tautomers that are capable of forming electronically stable anions, we should point out that the high-energy ZZ tautomer 14 which lies (in energy scale) about $1.13 \mathrm{eV}$ higher than the global minimum $\mathbf{1}$ is much less polar than would be expected for a system that contains two separated $\left(-\mathrm{NH}_{3}\right)^{+} /(-\mathrm{COO})^{-}$groups. Indeed, the net dipole moment of $\mathbf{1 4}$ is only $3.662 \mathrm{D}$ which is caused by the 
TABLE 2: Components of the Vertical Electron Binding Energies $D$ (in $\mathrm{cm}^{-1}$ ) of Dipole-Bound Anions Supported by Disulfide-Bridged Cystine Calculated with the 6-31 $+\mathrm{G}^{* *}+6(\mathrm{sp})$ Basis Sets ${ }^{a}$

\begin{tabular}{|c|c|c|c|c|c|c|}
\hline species & & $D^{\mathrm{KT}}$ & $\Delta D_{\text {ind }}^{S C F}$ & $\Delta D_{\mathrm{disp}}^{\mathrm{MP} 2}$ & $\Delta D_{\mathrm{no}-\mathrm{Misp}}^{\mathrm{MP}}$ & $\operatorname{sum}\left(D^{\mathrm{MP} 2}\right)$ \\
\hline \multirow[t]{2}{*}{$2^{-}$} & neutral geometry & 94 & 9 & 103 & -28 & $178(0.022)$ \\
\hline & anionic geometry & 105 & 10 & 115 & -31 & $199(0.025)$ \\
\hline \multirow[t]{2}{*}{$3^{-}$} & neutral geometry & 55 & 8 & 111 & -58 & $116(0.014)$ \\
\hline & anionic geometry & 61 & 9 & 120 & -63 & $127(0.016)$ \\
\hline \multirow[t]{2}{*}{$4^{-}$} & neutral geometry & 18 & 0 & 24 & -15 & $27(0.00335)$ \\
\hline & anionic geometry & 18 & 1 & 26 & -16 & $29(0.00359)$ \\
\hline \multirow[t]{2}{*}{$5^{-}$} & neutral geometry & 26 & 4 & 59 & -3 & $86(0.011)$ \\
\hline & anionic geometry & 33 & 5 & 73 & -4 & $107(0.013)$ \\
\hline \multirow[t]{2}{*}{$7^{-}$} & neutral geometry & 4 & 0 & 5 & -6 & $3(0.000372)$ \\
\hline & anionic geometry & 4 & 0 & 6 & -7 & $3(0.000372)$ \\
\hline \multirow[t]{2}{*}{$9^{-}$} & neutral geometry & 13 & 1 & 15 & -8 & $21(0.0026)$ \\
\hline & anionic geometry & 15 & 1 & 17 & -9 & $24(0.00297)$ \\
\hline \multirow[t]{2}{*}{$10^{-}$} & neutral geometry & 395 & 45 & 417 & -174 & $683(0.085)$ \\
\hline & anionic geometry & 514 & 70 & 533 & -224 & $893(0.111)$ \\
\hline \multirow[t]{2}{*}{$11^{-}$} & neutral geometry & 641 & 54 & 536 & -124 & $1107(0.137)$ \\
\hline & anionic geometry & 753 & 500 & 770 & -196 & $1827(0.226)$ \\
\hline \multirow[t]{2}{*}{$12^{-}$} & neutral geometry & 2228 & 519 & 1486 & -607 & $3626(0.449)$ \\
\hline & anionic geometry & 3163 & 647 & 1775 & -748 & $4837(0.599)$ \\
\hline \multirow[t]{2}{*}{$13^{-}$} & neutral geometry & 4117 & 690 & 1904 & -784 & $5927(0.735)$ \\
\hline & anionic geometry & 5496 & 838 & 2250 & -940 & $7644(0.947)$ \\
\hline \multirow[t]{2}{*}{$14^{-}$} & neutral geometry & 5 & 1 & 11 & -6 & $11(0.00136)$ \\
\hline & anionic geometry & 6 & 1 & 12 & -6 & $13(0.00161)$ \\
\hline $15^{-}$ & anionic geometry & 2610 & 608 & 1729 & -788 & $4159(0.516)$ \\
\hline $16^{-}$ & anionic geometry & 2531 & 545 & 1559 & -699 & $3936(0.488)$ \\
\hline $17^{-}$ & anionic geometry & 5349 & 819 & 2382 & -1146 & $7404(0.918)$ \\
\hline $18^{-}$ & anionic geometry & 3990 & 949 & 2881 & -1229 & $6591(0.817)$ \\
\hline $19^{-}$ & anionic geometry & 11349 & 1316 & 3493 & -1176 & 14982 (1.857) \\
\hline $20^{-}$ & anionic geometry & 12194 & 1379 & 3687 & -1333 & $15927(1.975)$ \\
\hline
\end{tabular}

${ }^{a}$ Final estimates of the vertical electron binding energies $D$ are also given in $\mathrm{eV}$ (see the values in parentheses).

cancellation of the local dipole moments due to the specific geometrical structure of this species (see Figures 2 and 5). In particular, the two $-\mathrm{NH}_{3}$ groups in $\mathbf{1 4}$ are oriented outward which causes the net dipole moment to be relatively small. Such a small dipole moment can be responsible only for relatively week excess electron binding and the resulting anion $\left(\mathbf{1 4}^{-}\right)$is indeed vertically electronically stable by only $0.00161 \mathrm{eV}$ (see Table 2). In each of the $\mathbf{1 0}^{-}-\mathbf{1 4}^{-}$anions, the extra electron is localized primarily outside the molecular framework on the positive side of the molecular dipole, as it has been previously observed for other dipole-bound anions (see Figure 5). ${ }^{24,25,31,35}$

As far as the issue of geometry relaxation upon excess electron attachment is concerned, the changes in geometry for $\mathbf{1 4}^{-}$(with respect to the corresponding neutral 14) are negligible (see Table 1S). The changes in geometry of the 10-13 species are larger although not significant. In particular, the geometry relaxations in those cases do not lead to qualitatively different structures and the observed changes in the bond lengths as well as in the valence and dihedral angles allow the final (i.e., anionic) structure to increase the dipole moment of the underlaying neutral molecular core (see Tables 1 and 1S).

3.4. Anions that Develop on the Anionic PES Due to the Significant Electron Binding Energies. As we mentioned in section 3.1., there could also exist other anionic cystine structures that correspond to local minima on the ground-state anionic potential energy surface but do not have corresponding geometrically stable neutral structures. Such minima would not correspond to any stable neutral isomer 1-14 described in section 3.1 but develop on the anionic PES due to the large vertical electron binding energy of the formed anion. A wellknown example of an analogous situation is the comparison of the neutral and anionic PES for the water dimer. For this hydrogen-bonded complex, one finds that there are two locally geometrically stable anions (i.e., CIS and TRANS conformers),
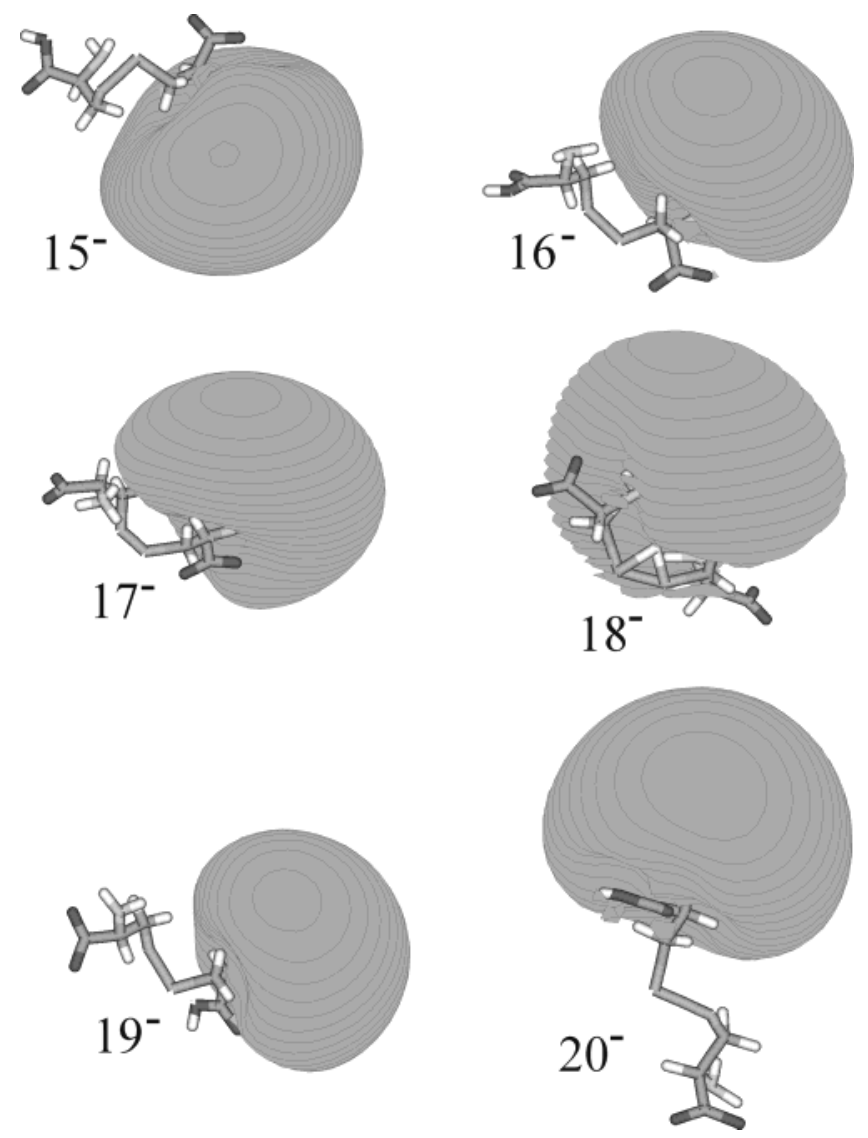

Figure 6. Singly occupied molecular orbital (SOMO) holding the excess electron in the ground electronic states of cystine-based anions $\mathbf{1 5}^{-}-\mathbf{2 0}^{-}$which do not correspond to any geometrically stable neutral parents (see section 3.4. for details).

whereas only one of them (TRANS) corresponds to the geometrically stable neutral parent. ${ }^{25}$ Therefore, the anionic "orphan" CIS may be treated as a species whose existence is caused by the large value of the electron binding energy characterizing the underlaying neutral core (in contrast to the TRANS daughter anion which corresponds to a geometrically similar neutral parent). The issue of the existence of such anionic orphans for cystine is the subject of this section.

While scanning the ground-state anionic PES for cystine, we found the total of 17 structures that correspond to local minima (see Figures 4-6, and Table 1S, in the Supporting Information). The discussion given in section 3.3 covers 11 of those structures (i.e., $\mathbf{2}^{-}, \mathbf{3}^{-}, \mathbf{4}^{-}, \mathbf{5}^{-}, \mathbf{7}^{-}, \mathbf{9}^{-}, 10^{-}, 11^{-}, 12^{-}, 13^{-}$, and $14^{-}$) for which we also found corresponding neutral parents. It turned out, however, that for 6 (out of 18) anionic structures we were unable to find any geometrically stable neutral structure that matches the existing anion. We numbered these species as $\mathbf{1 5}^{-}-$ $\mathbf{2 0}^{-}$and their relative energies (with respect to the global minimum 1) are given in Table 1, whereas the structures are depicted in Figures 3 (see also Table 1S, i the Supporting Information, where the geometrical coordinates for $\mathbf{1 5}^{-}-\mathbf{2 0}^{-}$ are collected). The singly occupied molecular orbitals holding the excess electron in $\mathbf{1 5}^{-}-\mathbf{2 0}^{-}$are depicted in Figure 6. As was observed for the other anions supported by cystine, the extra electron is localized on a diffuse orbital located primarily outside the molecular framework.

Most of these six orphan anions are relatively high in energy with respect to the neutral structure $\mathbf{1}$ that corresponds to the global minimum. In particular, the energies of $\mathbf{1 5}^{-}-\mathbf{1 8}^{-}$are higher than the energy of 1 by $0.558-0.998 \mathrm{eV}$ (see Table 1), 
whereas $\mathbf{1 9}^{-}$and $\mathbf{2 0}^{-}$are even higher and lie in the 1.529$1.583 \mathrm{eV}$ range.

Anions $\mathbf{1 9}^{-}$and $\mathbf{2 0}^{-}$are of special interest because their underlying neutral molecular core is extremely polar which is manifested by large values of the corresponding dipole moments (32.815 and 34.281 D for $\mathbf{1 9}$ and 20, respectively). It should be stressed though that $\mathbf{1 9}$ and $\mathbf{2 0}$ do not correspond to geometrically stable neutral species in this case but to the neutral structures obtained by removing excess electrons from $19^{-}$and $\mathbf{2 0}^{-}$without changing the geometries of those systems. Such large dipole moments are the result of the fact that the proton was transferred from the $-\mathrm{COOH}$ group belonging to one monomer to the $-\mathrm{NH}_{2}$ group belonging to another monomer. As a result, one monomer gathers the positive charge having both $-\mathrm{NH}_{3}{ }^{+}$(i.e., protonated $-\mathrm{NH}_{2}$ ) and $-\mathrm{COOH}$ (i.e., unchanged carboxyl group) while the other monomer collects the negative charge having both $-\mathrm{COO}^{-}$(i.e., deprotonated carboxyl group) and $-\mathrm{NH}_{2}$ (i.e., unchanged amino group).

It is important to notice that in the previously discussed ZC and $\mathrm{ZZ}$ species we observed the intra-monomer proton-transfer only. In the resulting systems (e.g., 11 and 12 of ZC nature, 13 and 14 of $\mathrm{ZZ}$ nature), the transfer of the proton occurred between the sites located very close to each other (i.e., neighboring $-\mathrm{COOH}$ and $-\mathrm{NH}_{2}$ functional groups). Therefore, the distance between $-\mathrm{NH}_{3}{ }^{+}$and $-\mathrm{COO}^{-}$in each resulting $\left(-\mathrm{NH}_{3}\right)^{+} /(-$ $\mathrm{COO})^{-}$pair was relatively small. In $\mathbf{1 9}^{-}$and $\mathbf{2 0}^{-}$, however, the proton is moved between distant $-\mathrm{COOH}$ and $-\mathrm{NH}_{2}$ functional groups which causes both (i) an increase of the total energy of the system and (ii) an increase of the polarity of the underlaying neutral molecule.

The large dipole moment of the neutral $\mathbf{1 9}$ and $\mathbf{2 0}$ (approaching 33-35 D) causes very strong excess electron binding. Indeed, the resulting vertical electron binding energies calculated for $\mathbf{1 9}^{-}$and $\mathbf{2 0}^{-}$(1.857 and $1.975 \mathrm{eV}$, respectively) are much larger than those obtained for all other anions studied in this work. However, the lowering of the total energy due to such a strong excess electron binding does not fully compensate for the energy increase caused by the separation of the charges discussed in the preceding paragraph. Therefore, $\mathbf{1 9}^{-}$and $\mathbf{2 0}^{-}$ are predicted to be highly thermodynamically unstable with respect to the species labeled $\mathbf{1}$ and a free electron (by 1.529 and $1.583 \mathrm{eV}$, respectively, see Table 1).

3.5. Analysis of the Electron Binding Energies of Cystine Anions. We now move on to the discussion of the vertical electron binding energies calculated for various cystine anions. The detailed results that are the subject of our analysis in this section are collected in Table 2. Since the contributions to the electron binding energies of the anions studied were often very small, we decided to use different energy units (i.e., wavenumbers instead of eVs) in both Table 2 and this section, which should make the discussion more convenient to the reader. However, to preserve consistency with other sections of this work, the total electron binding energies collected in Table 2 are also given in eVs.

The electron binding energy was partitioned into incremental contributions calculated at "successive" levels of theory (KT, $\mathrm{SCF}$, and MP2) as discussed in section 2, and the results for $\mathbf{2}^{-}-\mathbf{5}^{-}, \mathbf{7}^{-}$, and $\mathbf{9}^{-}-\mathbf{2 0}^{-}$are presented in Table 2 . The neutral species $\mathbf{1}, \mathbf{6}$, and $\mathbf{8}$ do not form electronically stable anions, so they are excluded from the analysis given in this section. In addition, we do not discuss the anion formed by $\mathbf{7}$ for which the final electron binding energy is estimated (at the MP2 level) to be only $3 \mathrm{~cm}^{-1}$, so we do not feel confident about our prediction of its electronical stability (including higher-than- second-order correlation effects would be necessary to give the more reliable estimate of $D$ in this case). For species $\mathbf{2}^{-}-\mathbf{5}^{-}$, and $\mathbf{9}^{-}-\mathbf{1 4}^{-}$the vertical electron binding energies were calculated for the equilibrium geometries of both the anion and its neutral parent, whereas for species $\mathbf{1 5}^{-}-\mathbf{2 0}^{-}$only the anionic geometry was considered (since the corresponding neutrals are not geometrically stable). However, to simplify the discussion, we limit it to the electron binding energies obtained for the equilibrium anionic geometries because they correspond to vertical electron detachment energies (VDEs) that are of more direct relevance to photoelectron measurements.

In the KT approximation, the electron binding energy results from the electrostatic and exchange interactions of the loosely bound electron with the SCF charge distribution of the neutral molecule. For all of the $\mathrm{CC}$ anions $\left(\mathbf{2}^{-}-\mathbf{5}^{-}, \mathbf{9}^{-}-\mathbf{1 0}^{-}\right.$, and $\left.\mathbf{1 5}^{-}\right)$, one $\mathrm{ZC}$ anion $\left(\mathbf{1 1}^{-}\right)$, and one $\mathrm{ZZ}$ anion $\left(\mathbf{1 4}^{-}\right)$, the $D^{\mathrm{KT}}$ values are relatively small: $<100 \mathrm{~cm}^{-1}$ for $\mathbf{3}^{-}, \mathbf{4}^{-}, \mathbf{5}^{-}, \mathbf{9}^{-}$, and $\mathbf{1 4}^{-}$, $105 \mathrm{~cm}^{-1}$ for $\mathbf{2}^{-}, 514 \mathrm{~cm}^{-1}$ for $\mathbf{1 0}^{-}$, and $753 \mathrm{~cm}^{-1}$ for $\mathbf{1 1}^{-}$(see Table 2). The $D^{\mathrm{KT}}$ values for all other anions exceed $2000 \mathrm{~cm}^{-1}$ and are the largest for $\mathbf{1 9}^{-}\left(11349 \mathrm{~cm}^{-1}\right)$ and $\mathbf{2 0}^{-}$(12 194 $\left.\mathrm{cm}^{-1}\right)$. In all cases, the $D^{\mathrm{KT}}$ terms are responsible for $31-77 \%$ of the total value of $D$ (see Table 2).

The SCF binding energies include orbital relaxation and thus take into account static polarization of the neutral molecule by the extra electron and the secondary effect of back-polarization. We found these contributions (which can be interpreted as orbital relaxation corrections to $D^{\mathrm{KT}}$, denoted $\Delta D_{\text {ind }}^{\mathrm{SCF}}$ ) to be responsible for $3-14 \%$ of the total $D$ (see Table 2).

The contribution denoted $\Delta D_{\text {disp }}^{\mathrm{MP} 2}$ results from dynamical correlation between the loosely bound electron and the electrons of the neutral molecule. This stabilization is caused by quantum mechanical charge fluctuations and is responsible for more than $50 \%$ of the total $D$ (for $\mathbf{2}^{-}, \mathbf{3}^{-}, \mathbf{4}^{-}, \mathbf{5}^{-}, \mathbf{9}^{-}, \mathbf{1 0}^{-}, \mathbf{1 1}^{-}$, and $\mathbf{1 4}^{-}$), and for $23-44 \%$ of the total $D$ (for $12^{-}, \mathbf{1 3}^{-}$, and $\mathbf{1 5}^{-}-\mathbf{2 0}^{-}$, see Table 2).

In addition to the dispersion interaction, other electron correlation factors may also affect the charge distribution (and multipole moments) of the neutral molecule and thus its electrostatic interaction with the extra electron. Such effects first appear at the MP2 level and are denoted $\Delta D_{\text {no-disp. }}^{\text {MP2 }}$. all of the cases at hand (excluding $\mathbf{6}$ which does not support a stable anionic state), MP2 electron correlation effects slightly reduce the dipole moment of the neutral system (see Table 1). Therefore, the value of $\Delta D_{\text {no-disp }}^{\mathrm{MP} 2}$ is destabilizing but always smaller than the corresponding (stabilizing) $\Delta D_{\text {disp }}^{\mathrm{MP}}$; thus, the total MP2 contribution to $D$ remains stabilizing due to the dominant role of the dispersion component.

Combining all of these contributions produces our final predictions for the vertical electron detachment energies that are presented in Table 2. One should keep in mind, however, that the VDEs given in this work (especially those characterizing weakly bound anions) might not be accurate enough because of the neglect of higher-than-second-order electron correlation contributions. We are aware of the fact that the electron binding energies presented in this work are likely underestimated and employing more sophisticated treatments (such as the coupledcluster method with single, double, and noniterative triple excitations) would be necessary to obtain more accurate results. Unfortunately, this was not possible due to the limited computer resources available at hand (the species we studied consists of 14 heavy atoms plus 12 hydrogen atoms).

Even though the vertical electron binding energies are very large for some of the anions (reaching $1.975 \mathrm{eV}$, see Table 2), it is important to notice that (i) the anions based on the most 
stable neutral isomers $\mathbf{1}-\mathbf{8}$ are either electronically unstable (1, 6, and 8) or weakly vertically bound (i.e., characterized by vertical electron detachment energies that do not exceed 0.025 $\mathrm{eV}$ ) and (ii) the anions that are strongly bound (by more than $0.45 \mathrm{eV})$, such as $12^{-}, \mathbf{1 3}^{-}, \mathbf{1 5}^{-}, \mathbf{1 6}^{-}, \mathbf{1 7}^{-}, \mathbf{1 8}^{-}, \mathbf{1 9}^{-}$, and $\mathbf{2 0}^{-}$, either correspond to structures that are not stationary points on the neutral PES (as $\mathbf{1 5}^{-} \mathbf{- 2 0 ^ { - }}$ ) or to high-energy neutral minima (as $12^{-}$and $\mathbf{1 3}^{-}$). Therefore, none of the anions studied in this work is adiabatically electronically stable with respect to the neutral structure $\mathbf{1}$ despite the fact that many negatively charged species based on the disulfide-bridged cystine possess large vertical electron binding energies. However, it seems likely that excess electron attachment to neutral cystine may result in forming anionic states that live long-enough to make their detection experimentally possible.

\section{Summary}

The possibility of electron binding to L,L-cystine in the gasphase was studied at the second-order Møller-Plesset perturbation theory level using $6-31+\mathrm{G}^{* *}+6(\mathrm{sp})$ basis sets. The lowestenergy isomer of the neutral disulfide-bridged L,L-cystine involves two canonical (rather than zwitterionic) cysteine monomers and exhibits an extended structure since it possesses only intra-monomer hydrogen bonds (i.e., no inter-monomer H-bonds). This isomer does not support an electronically stable anionic state although several other cystine isomers do. In particular, a total of 17 dipole-bound anionic species have been found with vertical electron detachment energies spanning a wide $0.0004-1.975 \mathrm{eV}$ range. Although most of the anions correspond to their neutral parents (from which they were derived), six of them were found to be geometrically stable only with the excess electron attached (i.e.,the corresponding neutral is geometrically unstable at this same geometry).

Acknowledgment. This work was supported by the Polish State Committee for Scientific Research (KBN) Grant No. DS/ 8371-4-0137-4 to P.S. and the NSF Grants CHE-9982420 and CHE-0240387 to J.S. The computer time provided by the Center for High Performance Computing at the University of Utah and the Academic Computer Center in Gdańsk (TASK) is also gratefully acknowledged.

Supporting Information Available: Table 1S containing the geometries for the neutral and anionic cystine structures at the Hartree-Fock/6-31+G**+5(sp) stationary points. This material is available free of charge via the Internet at http:// pubs.acs.org.

\section{References and Notes}

(1) Dai, S.; Schwendmayer, C.; Schürmann, P.; Ramaswamy, S.; Eklund, H. Science 2000, 287, 655.

(2) Lmoumène, E. H.; Comte, D.; Jacquot, J. P.; Houée-Levin, C. Biochemistry 2000, 39, 9295.
(3) Bergés, J.; Kassab, E.; Comte, D.; Adjadj, E.; Houée-Levin, C. J. Phys. Chem. A 1997, 101, 7809.

(4) Dahaoui, S.; Pichon-Pesme, V.; Howard, J. A. K., Lecomte, C. J. Phys. Chem. A 1999, 103, 6240.

(5) Ranganathan, D.; Lakshmi, C. Chem. Commun. 2001, 1250.

(6) Ross, S. A.; Burrows, C. J. Inorg. Chem. 1998, 37, 5358.

(7) Ranganathan, D.; Haridas, V.; Karle, I. L. J. Am. Chem. Soc. 1998, $120,2695$.

(8) Hameka, H. F.; Jensen, J. O.; Ong, K. K.; Samuels, A. C.; Vlahacos, C. P. J. Phys. Chem. A 1998, 102, 361.

(9) Carles, S.; Lecomte, F.; Schermann, J. P.; Desfrançois, Xu, S. Nilles, J. M.; Bowen, K. H.; Bergès, J.; Houée-Levin, C. J. Phys. Chem. A 2001, $105,5622$.

(10) Møller, C.; Plesset, M. S. Phys. Rev. 1934, 46, 618.

(11) Gutowski, M.; Skurski, P. J. Phys. Chem. B 1997, 101, 9143.

(12) Koopmans, T. Physica (Amsterdam) 1934, 1, 104.

(13) Skurski, P.; Gutowski, M.; Simons, J. Int. J. Quantum Chem. 2000 $80,1024$.

(14) McLean, A. D.; Chandler, G. S. J. Chem. Phys. 1980, 72, 5639.

(15) Krishnan, R.; Binkley, J. S.; Seeger, R.; Pople, J. A. J. Chem. Phys. 1980, 72,650 .

(16) Kendall, R. A.; Dunning, T. H., Jr.; Harrison, R. J. J. Chem. Phys. 1992, 96, 6796 .

(17) Schmidt, M. W.; Ruedenberg, K. J. Chem. Phys. 1979, 71, 3961.

(18) Gutowski, M.; Jordan, K. D.; Skurski, P. J. Phys. Chem. A 1998, $102,2624$.

(19) Frisch, M. J.; Trucks, G. W.; Schlegel, H. B.; Scuseria, G. E.; Robb, M. A.; Cheeseman, J. R.; Zakrzewski, V. G.; Montgomery, J. A., Jr.; Stratmann, R. E.; Burant, J. C.; Dapprich, S.; Millam, J. M.; Daniels, A. D.; Kudin, K. N.; Strain, M. C.; Farkas, O.; Tomasi, J.; Barone, V.; Cossi, M.; Cammi, R.; Mennucci, B.; Pomelli, C.; Adamo, C.; Clifford, S.; Ochterski, J.; Petersson, G. A.; Ayala, P. Y.; Cui, Q.; Morokuma, K.; Malick, D. K.; Rabuck, A. D.; Raghavachari, K.; Foresman, J. B.; Cioslowski, J.; Ortiz, J. V.; Stefanov, B. B.; Liu, G.; Liashenko, A.; Piskorz, P.; Komaromi, I.; Gomperts, R.; Martin, R. L.; Fox, D. J.; Keith, T.; Al-Laham, M. A.; Peng, C. Y.; Nanayakkara, A.; Gonzalez, C.; Challacombe, M.; Gill, P. M. W.; Johnson, B. G.; Chen, W.; Wong, M. W.; Andres, J. L.; Head-Gordon, M.; Replogle, E. S.; Pople, J. A. Gaussian 98, revision A.7; Gaussian, Inc.: Pittsburgh, PA, 1998.

(20) Schaftenaar, G.; Noordik, J. H. MOLDEN: a pre- and postprocessing program for molecular and electronic structures. J. Comput.-Aided Mol. Des. 2000, 14, 123.

(21) Skurski, P.; Simons, J. J. Chem. Phys. 2002, 116, 6118.

(22) Skurski, P.; Gutowski, M.; Simons, J. J. Chem. Phys. 1999, 110, 274.

(23) Skurski, P.; Gutowski, M.; Simons, J. J. Phys. Chem. A 1999, 103, 625 .

(24) Jordan, K. D.; Luken, W. J. Chem. Phys. 1976, 64, 2760.

(25) Gutowski, M.; Skurski, P. Recent Res. Dev. Phys. Chem. 1999, 3, 245.

(26) Gutowski, M.; Skurski, P.; Boldyrev, A. I.; Simons, J.; Jordan, K. D. Phys. Rev. A 1996, 54, 1906.

(27) Gutowski, M.; Skurski, P.; Simons, J.; Jordan, K. D. Int. J. Quantum Chem. 1997, 64, 183.

(28) Skurski, P.; Gutowski, M. J. Chem. Phys. 1998, 108, 6303.

(29) Elkadi, Y.; Adamowicz, L. Chem. Phys. Lett. 1996, 261, 507.

(30) Gutsev, G. L.; Bartlett, R. J. J. Chem. Phys. 1996, 105, 8785.

(31) Smith, D. M. A.; Smets, J.; Elkadi, Y.; Adamowicz, L. J. Chem. Phys. 1997, 107, 5788 .

(32) Gutsev, G. L.; Adamowicz, L. J. Phys. Chem. 1995, 99, 13412.

(33) Skurski, P.; Gutowski, M.; Simons, J. J. Chem. Phys. 2001, 114, 7443.

(34) Rak, J.; Skurski, P.; Gutowski, M. J. Chem. Phys. 2001, 114, 10673.

(35) Simons, J.; Skurski, P. The Roles of Electrostatics in Forming Molecular Anions and Dianions. In Theoretical Prospects of Negative Ions; Kalcher, J., Ed.; Research Signpost: Trivandrum, 2002. 